\title{
GERENCIAMENTO DA ÁGUA COM A INTERNET DAS COISAS (IOT): UMA APLICAÇÃO EM PLANTAS DE SANEAMENTO
}

WATER MANAGEMENT WITH THE INTERNET OF THINGS (IOT): AN APPLICATION IN SEWERAGE PLANTS

\author{
ALAIDE BARBOSA MARTINS* \\ CLÁUDIA CRISTINA RIOS CAXIAS DA COSTA** \\ MARCELO TEIXEIRA DE AZEVEDO*** \\ SEGIO TAKEO KOFUJI"***
}

\begin{abstract}
R E S U M O
Considerando que a água apresenta importância sanitária e econômica, verifica-se que os sistemas hídricos automatizados e as diversas plantas de serviços críticos estão cada vez mais se modernizando e integrando as infraestruturas existentes, em nuvens de gerenciamento de dados. Em função do ganho de produtividade e investimento mais baixos, a aplicação de tecnologias de Internet das Coisas (IOT) em diversas áreas torna-se uma realidade. Com o aumento da complexidade dos sistemas hídricos, os riscos de segurança tendem a aumentar, porém os ganhos em gerenciamento e qualidade do tratamento da água podem trazer uma maior confiabilidade ao sistema. Partindo-se desta problemática e com o atual desenvolvimento tecnológico em que se encontra a área de IoT, é aqui proposto um estudo de aplicação desta tecnologia em plantas de saneamento.
\end{abstract}

PALAVRAS-CHAVE: Internet das Coisas, Segurança da informação e do processo, plantas de saneamento.
A B S T R A C T

Whereas the water presents health and economic importance, it turns out that the automated water systems and the various plants of critical services are increasingly modernizing and integrating existing infrastructures, in clouds of data management. In terms of productivity gains and lower investment, the application of technologies of Internet of things (IOT) in several areas becomes a reality. With the increasing complexity of water systems, security risks tend to increase, but the gains in management and quality of water treatment can bring greater reliability to the system. Assuming this problem and with the current technological development in the area of IoT, here is proposed a study of the application of this technology in sanitation plants.

KEYWORDS: Internet of things, Information security, Sanitation plants process.

\footnotetext{
"Doutora em Engenharia Elétrica - Sistemas Eletrônicos pela Universidade de São Paulo (POLI-USP), Pós-Doutoranda em Engenharia Elétrica pela Universidade de São Paulo (POLI-

USP), São Paulo-SP, Brasil, e Docente na Faculdade Dom Pedro II de Tecnologia, Salvador-Bahia, Brasil. Correo electrónico: alaide@uspbr

** Doutora em Geografia - Tratamento da Informação Espacial pela Pontifícia Universidade Católica de Minas Gerais (PUC-Minas) e Docente na Faculdade Dom Pedro II de Tecnologia, Salvador-Bahia, Brasil. Correo electrónico: riosdacosta@gmail.com

*** Doutor em Engenharia Elétrica - Sistemas Eletrônicos pela Universidade de São Paulo (POLI-USP) e Pós-Doutorando em Engenharia Elétrica pela Universidade de São Paulo (POLI-USP), São Paulo-SP, Brasil. Correo electrónico: mdeazevedo@usp.br

${ }^{* * * *}$ Doutor em Engenharia Elétrica - Professor Doutor RIDP da Escola Politécnica da Universidade de São Paulo (POLI-USP), São Paulo-SP, Brasil. Correo electrónico: kofuji@usp.br
} 


\section{INTRODUÇÃO}

No Brasil, nas últimas duas décadas, o uso das geotecnologias como ferramenta de apoio à tomada de decisões, tornou-se uma tendência. As geotecnologias se traduzem como o conjunto de tecnologias para coleta, processamento, análise e disponibilização de informação com referência geográfica, compostas por soluções em hardware, software e peopleware que juntos se constituem em poderosas ferramentas para inferências, classificações e julgamentos. Hodiernamente, constata-se a importância do uso das geotecnologias, bem como a irreversibilidade do uso destas nos estudos dos espaços geográficos. Nesses denota-se o acesso aos recursos hídricos, como temática recorrentemente tratada na perspectiva urbano-espacial. O controle da disponibilidade e qualidade da água, entendida como necessidade básica, carece de estudos cada vez mais atrelados às geotecnologias, visando o monitoramento e solução para as perdas e vazamentos existentes na rede de distribuição hídrica, que atende a sociedade como um todo.

No âmbito da gestão pública, Duarte (2010) atenta para o uso das geotecnologias, no tocante ao planejamento e também melhor atendimento aos cidadãos, que através de mapas e memoriais descritivos podem rapidamente acessar conteúdos de interesse, a exemplo dos serviços de distribuição de água. Ante a crescente demanda dos recursos hídricos e de sua premente escassez, faz-se necessário aprimorar o planejamento de manutenção das redes de abastecimento, bem como agilidade e acesso, no que tange ao reparo nas mesmas.

Sobre o gerenciamento das redes de abastecimento de água:

O setor de saneamento básico no Brasil vem apresentando ao longo das últimas décadas altos índices de perdas de água, que devem ser combatidos através de um conjunto de ações que incluam um melhor planejamento, operação e manutenção dos elementos que o constituem. Nesse sentido, os recursos disponíveis nos Sistemas de Geoinformação permitem efetuar análises e simulações de operação e manutenção em sistemas de abastecimento de água, além de possibilitar, em um único ambiente, o intercâmbio de informações entre o cadastro técnico, responsável pelas redes de infraestrutura, e o cadastro comercial, responsável pe- 
las informações relativas aos consumidores. (Borges,Sá \& Gomes, 2004)

Nos últimos anos, o número de plantas de tratamento de água e esgoto - que estão automatizadas com instrumentos de monitoramento do processo e com sistemas de controles - teve considerável incremento. Entretanto, a preocupação em segurança da informação e a análise dos riscos do processo ainda são incipientes nas plantas brasileiras.

Os ambientes de automação industrial estão agregados às tecnologias de comunicação que permitem à mobilidade e convergência das geotecnologias ligadas a informação. Esse cenário tornou estas redes susceptíveis a ataques colocando em risco o abastecimento hídrico, sobremaneira nos grandes centros urbanos. Entretanto, qualquer interferência nos processos de tratamento de água pode causar prejuízos enormes à sociedade. Cordão et al. (2013) atentam para o fato da aplicação da modelagem e otimização da disposição espacial de unidades de reservação ${ }^{1}$ que constituem os Sistemas Urbanos de Distribuição de Água (SUDA), sob a perspectiva do apoio à decisão no planejamento dos

' A reservação é empregada para o acúmulo da água, visando atender a variação do consumo, a manter uma pressão mínima ou constante na sistemas de abastecimento e saneamento.

O objetivo central desta pesquisa é desenvolver uma análise da aplicação da tecnologia de Internet das Coisas (IoT) em plantas de saneamentos, avaliando desde dos ganhos da aplicação para o processo, bem como o desenvolvimento de uma análise de risco com avaliação qualitativa e quantitativa, que valide ou não a aplicação de uma arquitetura de IoT, tendo como base métodos existentes para análise de risco. Ao mesmo tempo, pretende-se com o aprimoramento das modelagens matemáticas, a identificação do ganho de eficiência, em face de utilização da internet das coisas na operação de plantas de saneamento.

A Internet das Coisas pode ser entendida, em um de seus aspectos, como uma inovação tecnológica, que tem como base uma série de equipamentos e instrumentos de campo inteligentes e interligados que desenham um cenário totalmente automatizado, com respostas rápidas e decisões próprias. Segundo Schwab (2016), as sociedades modernas vivem no limiar de uma quarta revolução industrial, a chamada revolução industrial baseada no conceito de sistemas Cyber-Physical

rede, bem como atender demandas de emergências, em casos de incêndios, ruptura ou desvio de rede, etc. 
Systems. Essa quarta revolução industrial interfere basicamente nos pilares físico, digital e biológico, tendo impacto na economia, negócios, sociedade, indivíduo e governo.

As automações das plantas contribuem para o aumento do volume de negócios, ao mesmo tempo em que implicam na redução da mão de obra assalariada. Entretanto, proporcionam uma crescente complexidade de instalações e processos, facilitados pelo contínuo desenvolvimento de projetos sofisticados de controle e das geotecnologias que se propõem a facilitar tais demandas. Nessa perspectiva, em função desta série de mudanças sucessivas, origina-se um cenário emergente e com ele, a necessidade de um novo tipo de gestão de segurança de processos atrelados as plantas de saneamento.

Ao longo dos anos, estes sistemas migraram por diversas soluções tecnológicas tendo por proposta o desenvolvimento da análise dos impactos, bem como a possibilidade de novos avanços, em um curto espaço de tempo.

Além de eventos físicos, as plantas de tratamento de água estão sujeitas a ataques cibernéticos que podem, por exemplo, interferir diretamente no sistema de dosagem de um determinado produto químico, contaminando a água a ser distribuída. Além dessa possibilidade, vislumbra-se a ocorrência de vazamentos de determinado produto, a alteração de vazões de bombeamento de uma rede específica de distribuição ou mesmo - em casos extremos - o transbordamento de reservatório.

Segundo Kroll (2006), a análise da vulnerabilidade das plantas de tratamento de água tende, cada vez mais, a fazer parte da preocupação da sociedade. Contudo, observa-se que realizar atividades de quantificação e qualificação - atreladas a vulnerabilidade de uma planta de abastecimento - não é uma tarefa tão fácil. Existem muitos métodos e ferramentas integradas disponíveis, nada triviais, que possibilitam quantificar a análise de risco e de falhas, tanto no cenário de segurança da informação como na própria segurança do processo. Com recursos disponíveis e integração as centrais de cidades inteligentes, as respostas aos incidentes, em situações de vulnerabilidades podem ser mais rápidas e eficientes.

Diante do exposto, justifica-se este trabalho, que apresentará a avaliação da aplicabilidade prática da Internet das Coisas, em plantas de saneamento com ênfase nos seus ganhos e riscos fomentando assim, a análise dos processos envolvidos, em tempo real. 


\section{A INTERNET DAS COISAS (IOT): CONCEITO E PARTICULARIDADES}

A Internet das Coisas (IoT) é genericamente compreendida, como o sistema de dispositivos conectados à Internet, que fornecem as empresas de Utilities $^{2}$, dados sobre como as pessoas as utilizam. As informações geográficas, no que tange a aplicabilidade dessa nova tecnologia são fundamentais. A difusão da IoT fomentará a essas empresas, uma nova fonte de informações sobre como fornecer melhores serviços aos seus usuários, além de possibilitar minimização de gastos de cunho operacional.

Ao relacionar nessa pesquisa a área de saneamento a IoT, temática relativamente nova e incipiente em pesquisas, recorreu-se a autores e projetos consolidados internacionalmente que contribuíram, sobremaneira, para o desenvolvimento do estudo.

Sun et al. (2012) já abordavam a Internet das Coisas (IoT) na computação em nuvens, fazendo menção ao monitoramento e alarme de sistemas. Na mesma época, foram empreendidos estudos sobre arquiteturas de IoT (Hompel et al., 2012) e aplicação de
Internet das Coisas em construções sustentáveis com aplicações totalmente automatizadas em ambientes residenciais (Luo et al. 2012).

Oren e Stroh (2013) apresentam um modelo matemático para detectar vazamentos em tempo real no consumo doméstico da água. O modelo utiliza dados que são medidos a partir de um hidrômetro, empregando dois critérios simultaneamente, a saber: desvio do consumo médio e comparação de consumo de água durante um período de tempo. A simulação do modelo de consumo doméstico foi implementado em um equipamento construído pelos autores, denominado Antileaks, dispositivo que transfere as informações analógicas de consumo para um formato digital e em tempo real.

Pietrabissa et al. (2015), idealizaram um sistema de gestão da informação para o novo paradigma da fábrica do futuro, enquanto Muhammad (2015), utilizaram técnicas de machine learning $^{3}$ com o objetivo de classificar a qualidade da água, descobrindo assim, o melhor modelo para realizar esta classificação. Para isto, foram aplicados cinco modelos: Naive Bayes, Conjunctive Rule, J48, Kstar e Bagging. Nos testes propostos pelos

\footnotetext{
3 O aprendizado de máquina é uma área da ciência da computação que tem como objetivo responder a uma simples questão: como ensinar máquinas a aprender?

\footnotetext{
2 Água (armazenamento, tratamento e distribuição), energia elétrica (geração, transmissão e distribuição), gás (armazenamento e distribuição), esgoto (coleta, tratamento e descarte) e serviços de limpeza pública.
} 
autores, o algoritmo Kstar foi o que teve melhor precisão.

Mounce (2015) partiram da assertiva de que um terço dos serviços públicos, em todo o mundo, aferem uma perda de quase $40 \%$ da água limpa devido a vazamentos. Esta perda poderia ser minimizada pela utilização de um sistema de água inteligente, capaz de coletar informações importantes, por meio da IoT, utilizando sensores localizados ao longo da rede hídrica. Esses dados, processados por sistemas de Inteligência Artificial (AI) e manipulados por grandes redes de dados (Big Data), oferecem um plano de monitoramento e solução para as perdas e vazamentos. Conceitualmente foi proposta a análise de perdas em um ambiente controlado, utilizando-se técnicas de machine learning para prever os vazamentos, tendo como parâmetro os dados históricos de vazão e pressão.

Soldatos, Gusmeroli, Malo e Di Orio (2016) partem do princípio de que a fábrica do futuro é impulsionada pela flexibilidade da fabricação e respostas rápidas, as necessidades do mercado. A pesquisa dos autores ainda contempla iniciativas, tecnologias IoT e aplicações relacionadas as tecnologias de internet do futuro, entre elas o projeto

${ }_{4}^{4}$ Projeto europeu que objetivou estudar e apresentar dez casos experimentais da Fábrica do Futuro, levando-se em consideração a evolução
Future Internet in the Manufacturing industries (FITMAN) ${ }^{4}$. Um dos objetivos do estudo foi demonstrar como a IoT pode transformar a manufatura utilizando tecnologias emergentes e para isto, ilustrou implantações possíveis para a fábrica do futuro. Recentemente Jenkins (2016) abordou a aplicação de Internet das Coisas em Plantas de Água, explanando com riqueza de detalhes, possíveis aplicações nesse campo.

Conforme Fiaschetti, Pietrabissa e Delli Priscoli (2015) norteados pela ideia de que o novo modelo incorporado pela fábrica do futuro produz uma quantidade considerável de informações para o operador, alertam para a utilização das ferramentas tradicionais disponíveis, que no trato com o gerenciamento das informações, torna-se um trabalho pouco efetivo para tomada de decisão. Partindo desta problematização, os autores concluem que são necessárias novas tecnologias para lidar com processos fabril inovadores. O estudo demonstra ainda uma solução de arquitetura inovadora baseado na utilização e integração em ICT - Information and Communications Technology, tendo como base o framework Future Internet, especificamente o projeto PLATINO. Em uma tomobilístico, aeronáutica, têxtil, etc. 
abordagem mais recente, Meola (2016) apresentou a aplicabilidade da Internet das Coisas em plantas de utilidades de gás, energia elétrica e água ${ }^{5}$.

\section{A IOT E AS VULNERA- BILIDADES EM PLANTAS DE SANEAMENTO: A HI- PÓTESE TRABALHADA}

A hipótese pleiteada nessa pesquisa faz referência as inúmeras vulnerabilidades existentes em plantas de saneamento, especialmente com aplicações de IoT. A partir deste contexto, este trabalho propõe o desenvolvimento de um modelo de análise de risco próprio para plantas de saneamento, que possa validar uma arquitetura de monitoramento on-line com instrumentos e rede de automação segura. Para tal intuito, aplicou-se técnicas, e ferramentas tradicionais que possibilitaram realizar a otimização combinatória para avaliação dos possíveis comportamentos dos sistemas, fornecendo respostas necessárias a metodologia de análise de falha.

Enfatizam Mckinsey (2016), I-scoop (2016), BCG (2015) e Gartner (2016) que a indústria de Utilities ainda não sabe ao certo os efeitos da aplicação em larga escala da IoT, portanto, esse

${ }^{5} \mathrm{O}$ projeto PLATINO concebeu um framework para as fábricas do futuro. Sua modelagem se adequa a proposta desse estudo, no tocante a trabalho torna-se uma contribuição para o cenário da Indústria de Utilities, cujas transformações digitais se avolumam. Ao analisar todo o ecossistema da indústria de Utilities, faz-se necessário quebrar a cadeia para encontrar os desafios e oportunidade por cada elemento. A Accenture (2016) resumiu a cadeia de suprimentos da Indústria de Utilities em três grandes grupos, denotados na Figura 1: Supply (onde são gerados os recursos); Network (onde ocorre a transmissão e distribuição) e o Retail (consumidores).

Nas próximas seções será discutida a questão da transformação digital na Indústria de Utilities, especificamente, em uma estação de tratamento de água. $\mathrm{O}$ foco do estudo direciona-se a camada de supply, na qual é realizada a captação da água bruta para posterior tratamento.

Na grande área de Utilities destaca-se a aplicabilidade da plataforma para transformação digital energética denominada FINESCE (Future Internet Smart Utility Services) Smart Energy Plataform, que serviu como base para a definição da nova plataforma para as plantas de saneamento. O projeto em questão - através de exemplos práticos - mostra a importância em se tra

aplicabilidade da loT nas plantas de tratamento da água. 
Figura 1. Digital Utility

\section{The Digital Utility: Transforming for value and growth}

Pursuing digital transformation across the value chain can be the most effective and cost-efficient way to address business challenges and achieve sustainable growth.

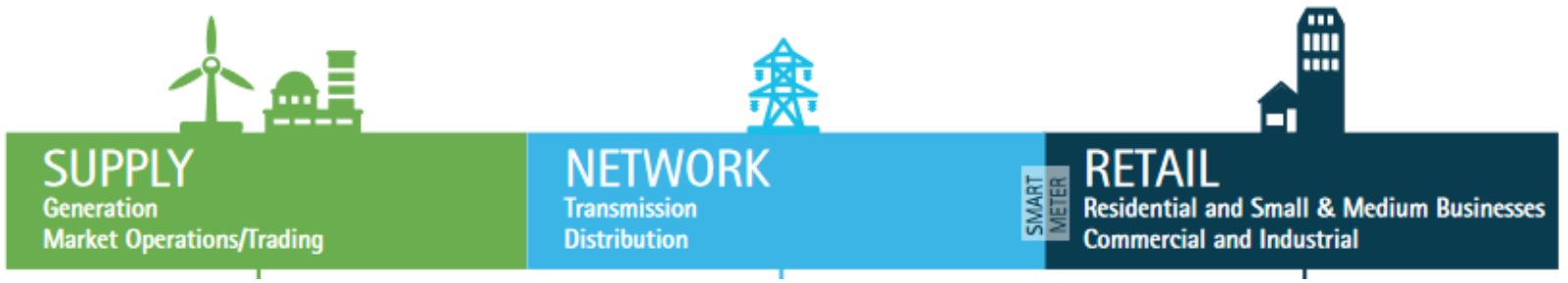

Fonte: Accenture (2016).

Figura 2. Terni - Smart Grid \& Energy Marketplace

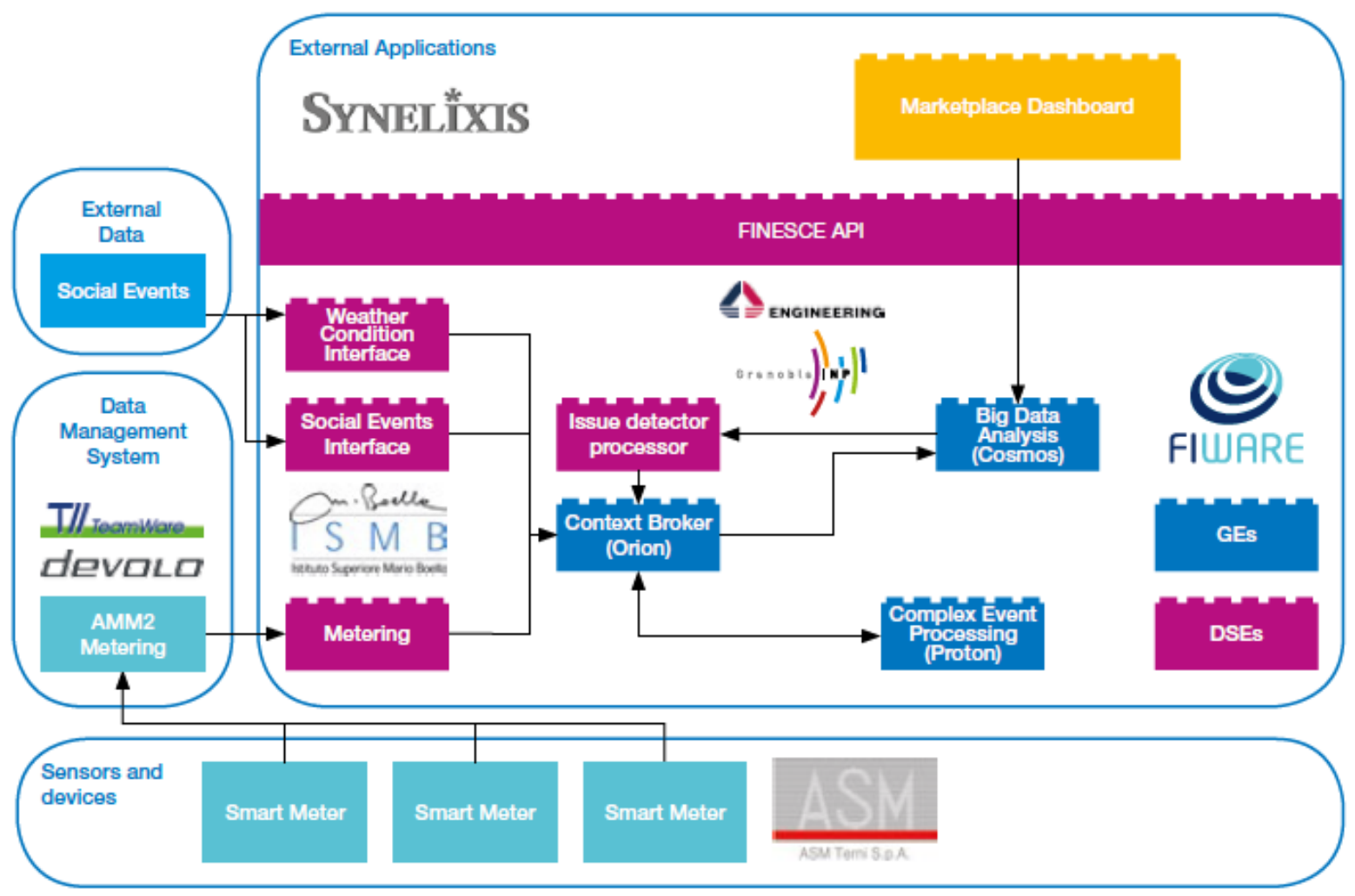

Fonte: FINESCE (2016).

balhar com soluções digitalizadas, baseadas em nuvem aplicáveis ao setor de energia, visando o futuro.
A Utility 4.0 - novos serviços de energia ativados por plataformas de Tecnologia da Informação e Comunicação 
(TIC) - trata da implementação de sete diferentes sites na Europa, sendo que cada um destes mostra a viabilidade de utilização do Fiware para uma solução específica de Smart Energy. Dentre os projetos destaca-se, o Terni - Smart Grid \& Energy Marketplace (FIG. 2). Atenta-se para a interface DSE (Domain Specific Enablers) para condição do tempo, na qual são coletados dados sobre a previsão a cada 5 minutos, enviados ao ORION Context Broker. Já a interface DSE de "eventos sociais" é um método em que um agente externo pode prover informações sobre fenômenos que podem afetar a produção e o consumo de energia, tais como: concertos, partida de futebol e eventos sociais genéricos. O GE Big Data (COSMOS), o Context Broker GE (ORION) e o Complex Event Processing GE (PROTON) são integrados como parte central da arquitetura.

Essa pesquisa também contemplou o projeto da Finodex (Future Internet Open Data Expansion), iniciativa financiada pela Comissão Europeia, cujo objetivo é fomentar a criação de produtos e serviços inovadores baseados em dados abertos. Neste contexto, o projeto conta com a estrutura do FIWARE (utilizando a sua plataforma aberta para oferecer novos serviços e aplicativos) com a empresa Adevice, de Sevilha, na Espanha (que fornece hardware industrial adequado para aplicações FIWARE, tais como: gateways e devices para coleta de dados).

O objetivo desta integração é a implementação de um projeto-piloto chamado Smart Fountain, que permite a medição de parâmetros de qualidade da água em fontes municipais e piscinas da cidade de Sevilha, sendo um exemplo de combinação dos requisitos da Indústria 4.0 com cidades inteligentes. Na Figura 3 é possível observar a arquitetura definida por Cabezas (2016).

O conceito de água não faturada, como vulnerabilidade nos processos que envolvem o tratamento de uma planta hídrica, é de suma importância para a compreensão da metodologia aplicada nesse estudo. Entende-se por água não faturada todo o volume perdido, devido a existência de vazamentos na rede de distribuição, tendo por origem variáveis tais como: idade dos tubos, envelhecimento e qualidade do material de revestimento desses, má instalação, tráfego de veículos, variações de pressão, dentre outros.

Melhorar modelos existentes, através de combinações de técnicas de modelagens visando desenvolver, bem como implementar, um modelo de análise de risco otimizado, tornou-se também, objetivo desse estudo. Aqui contempla-se a complexidade de plantas 
de saneamento permitindo a intrato com a segurança do processo e teração do ambiente automatizado, do tráfego de informações sobre esse. com ênfase em melhores práticas no

Figura 3. Arquitetura de comunicação FINODEX Sevilha

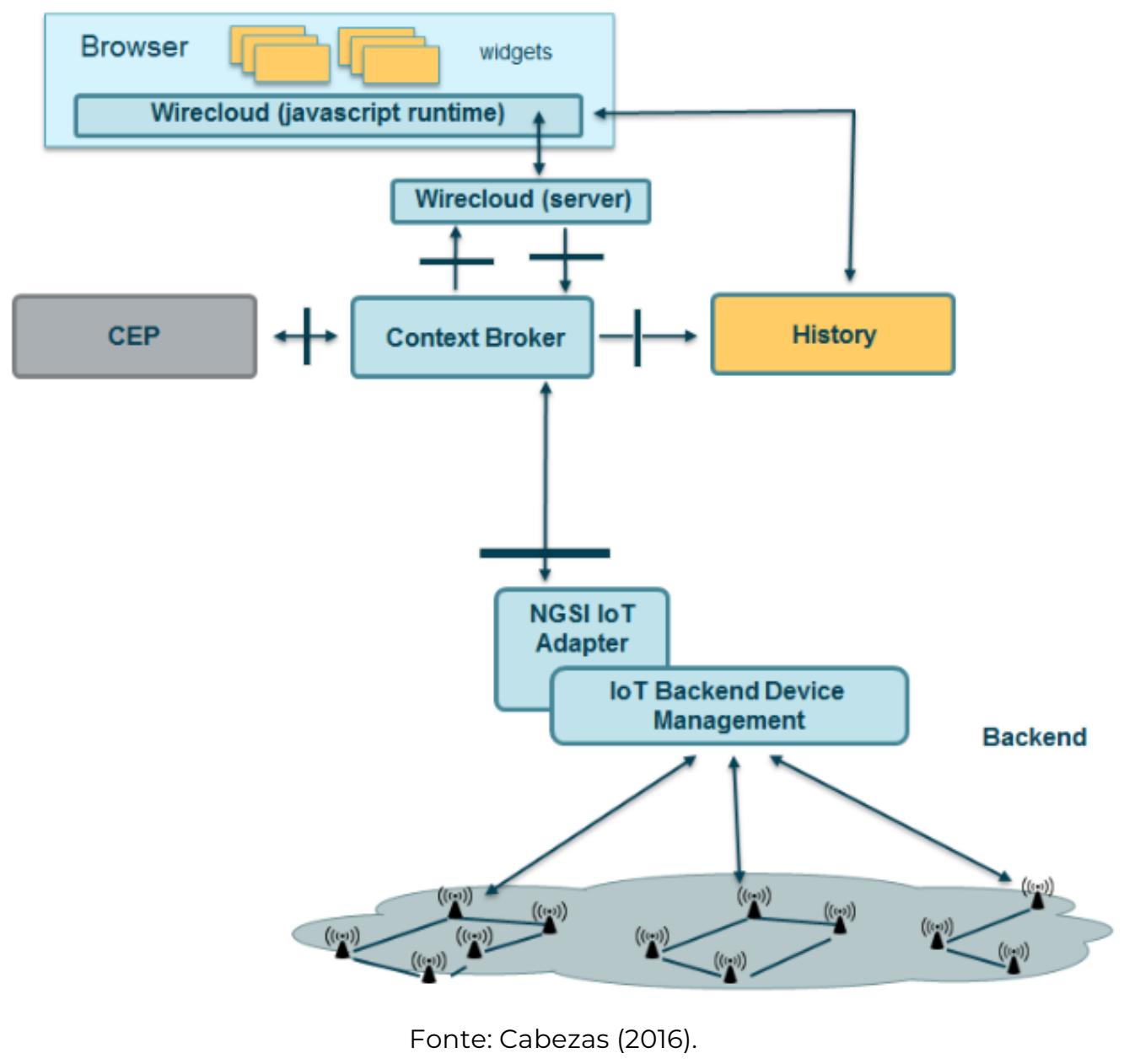

\section{ITINERÁRIO METODO- LÓGICO E RESULTADOS DA PESQUISA}

Para a composição desse estudo, empregou-se como procedimento metodológico, a revisão da literatura, problematização, bem como a análise da literatura específica sobre a IoT e suas aplicabilidades em plantas de saneamento. A metodologia utilizada neste trabalho foi o método científico da inferência, aliado a coleta de dados mediante revisão bibliográfica sobre o tema e utilização de normas. O planejamento da pesquisa contemplou duas etapas interligadas: o estudo teórico e documental, e o estudo empírico com desenvolvimento da simulação de dados reais, ambos com foco em plantas de saneamento. 
Elementos importantes desse estudo foram facultados pela utilização do software gratuito disponibilizado pela Environmental Protection Agency (EPA), o EPANET associado a outras ferramentas evidenciadas por modelos de sistema, simulação de coeficientes e resposta discretizada, conforme observa-se nas figuras 6, 7 e 8. A aplicação do conceito de IoT foi realizada em uma planta de tratamento de água, com base no processo convencional de tratamento, como demonstra a Figura 4.

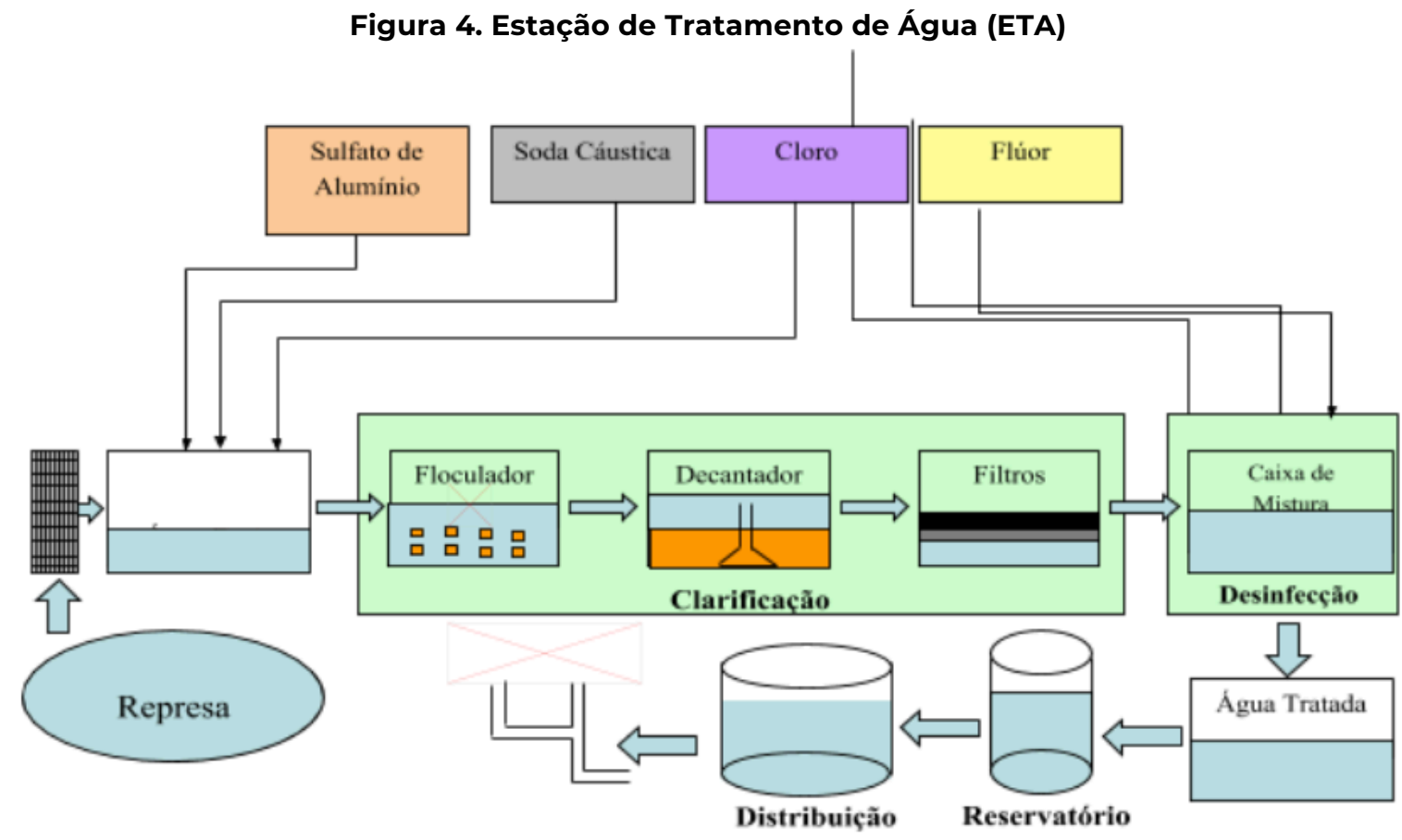

Fonte: Elaborada pelos autores.

Atenta-se que o controle da qualidade de água é de responsabilidade da empresa que realiza o serviço de tratamento, juntamente com a vigilância sanitária e a agência reguladora. Esse controle é realizado por meio de análises físico-química e microbiológicas das variáveis a seguir:

- $\mathrm{pH}$

- sabor e odor;

- turbidez;
- cor;

- dureza;

- concentração de: cloro, flúor, alumínio, ferro, manganês, entre outros;

- determinação de OD.

O novo modelo tendo como base a arquitetura de comunicação FINODEX foi aplicado, conforme as Figura 5, 6 e 7. 
Figura 5. Modelo IoT para Saneamento

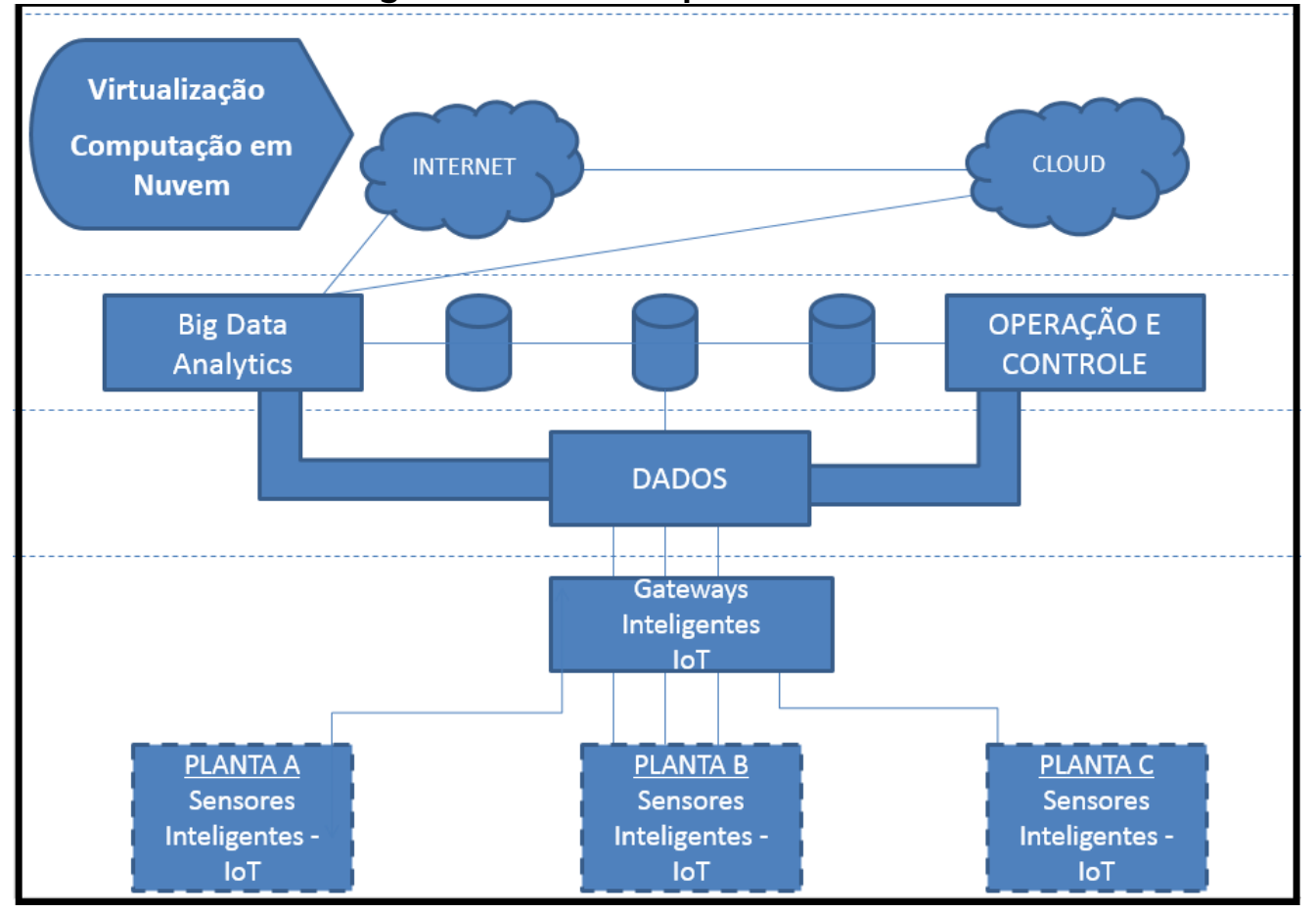

Fonte: Elaborado pelos autores.

Figura 6. Processo Analítico - SCADA para Saneamento

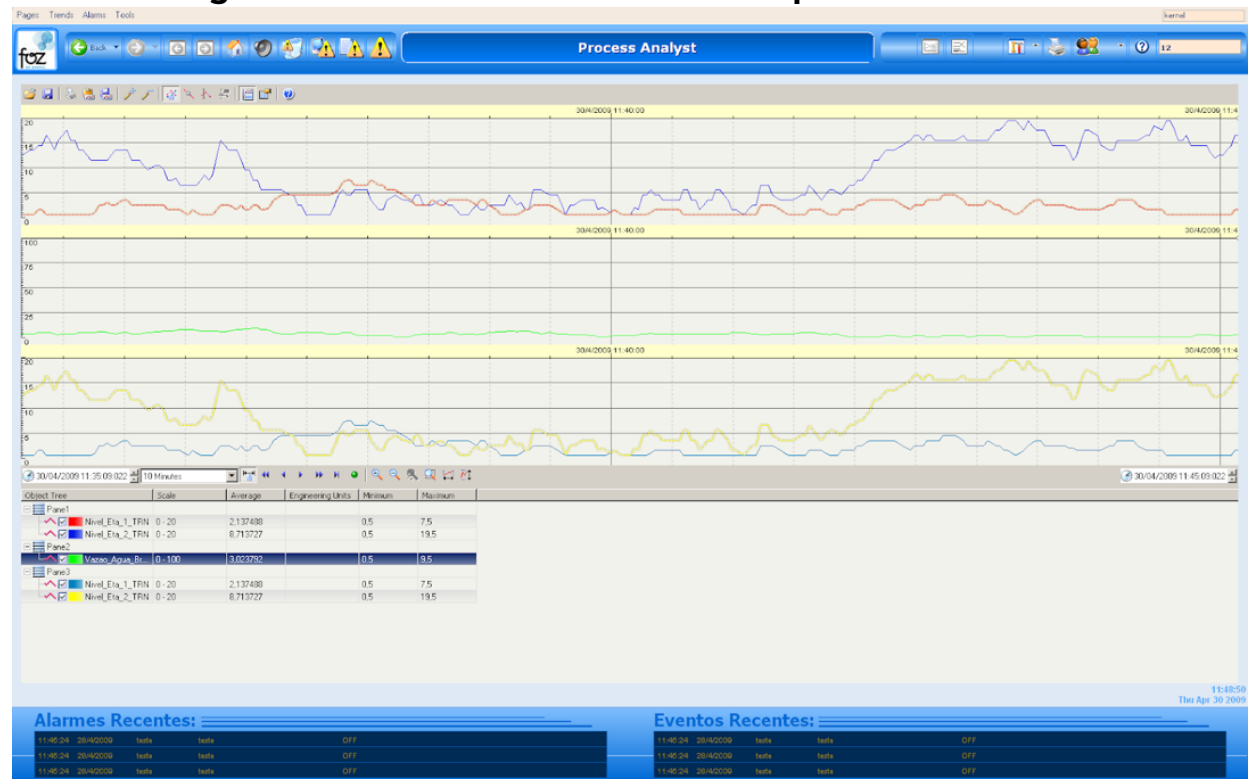

Fonte: Elaborado pelos autores. 


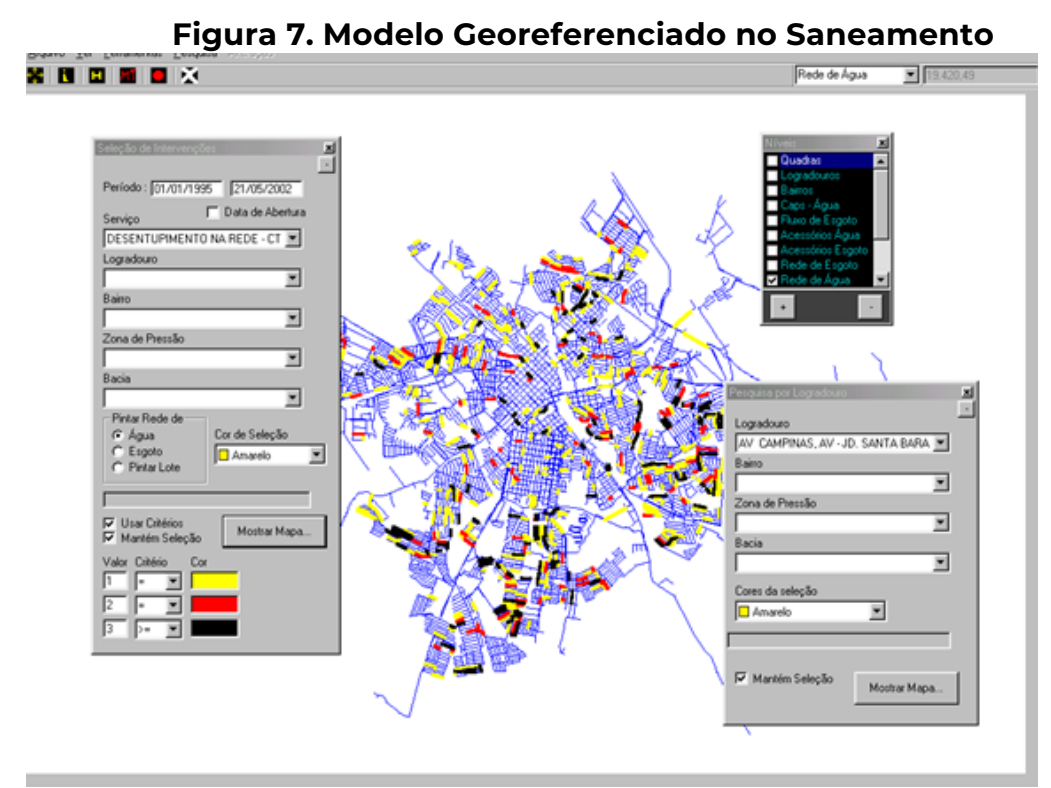

Fonte: Próprio autor.

Conforme a análise quantitativa, os dados do processos foram ajustados em um modelo linear (reta) nas base de teste (pontos): PH_D x PH_S, para posterior ajuste na produção:

Figura 8. Regressao Linear PH_D x PH_S

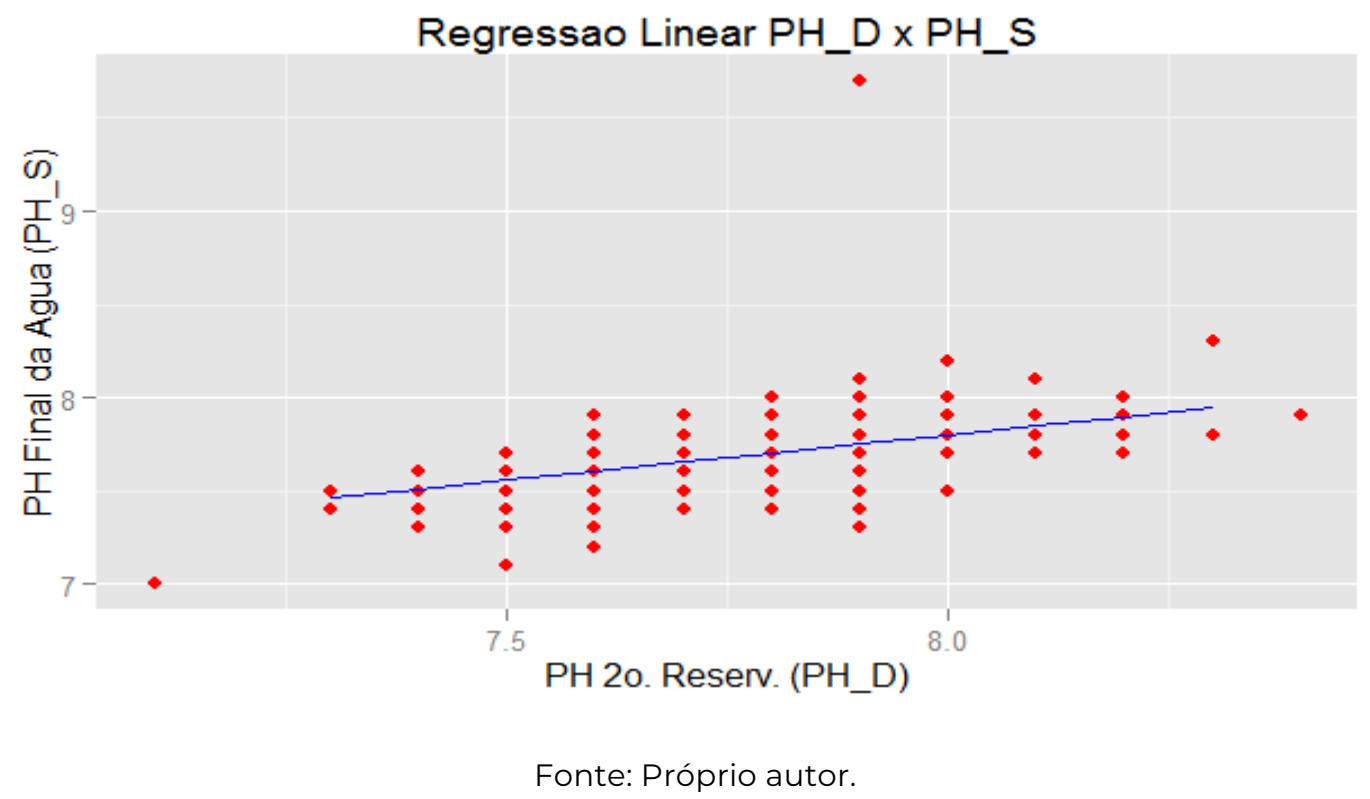

Apesar de os dados variarem um pouco em relação à média, a reta do modelo está bem centrada, indicando que, na média, o PH final da água (PH_S) 
pode vir a ser previsto por um modelo linear que tem como base os valores de $P H$ do segundo reservatório (PH_D).

Apurou-se que os ganhos observados na utilização desse novo modelo, comparado com a arquitetura tradicional, foram:

1. Utilização de dados históricos para prever necessidades, colaborando com toda a cadeia de suprimento para atender à demanda pelo recurso;

2. A partir de um local remoto, gerenciar as fontes de água e ter o controle e o uso definidos por processos automatizados;

3. Integrar-se a fontes de dados não relacionadas à água, tais como: governo, pesquisa e indústria, de uma maneira geral, para acompanhar os esforços de conservação e melhoria contínua da operação existente;

4. Por meio do uso de painéis de controle, aumentar a visibilidade das instalações de ativos e melhorar o desempenho e análise de dados;

5. Utilização de dados em tempo real sobre eventos climáticos, visando antecipar as mudanças na disponibilidade ou necessidades de recursos;

6. Propiciar aos consumidores um maior controle e visibilidade do consumo, bem como dos recursos residenciais e urbanos, para direcionar esforços na conservação do recurso;

7. Utilização dos dados para descobrir melhorias nos procedimentos e, assim, proporcionar um melhor atendimento aos cidadãos;

8. Melhoria na precisão das leituras dos medidores de água com maior frequência.

\section{CONSIDERAÇÕES FI- NAIS}

A incorporação de novas tecnologias, a exemplo da Internet das Coisas, no que tange ao sistema de abastecimento de água permitirá, em larga escala, que as empresas gestoras da utilitie obtenham dados durante todo o processo, que podem ser analisados e processados em tempo real, minimizando custos operacionais consideráveis.

Para o empreendimento desse estudo ficou notória a carência de pesquisas que contemplem a segurança de processo (safety) e segurança da informação (security), principalmente em plantas de tratamento de água. Aliadas a isso, a revisão da literatura aponta a ausência de aplicação de tecnologia de análise de risco, verificação de falhas e de maiores investimentos em segurança do processo para plan- 
tas de saneamento. A alta vulnerabilidade das plantas de tratamento hídrico, bem como o impacto que essas podem causar a sociedade na ocorrência de ataques ou erros operacionais foi a motivação para o desenvolvimento desse trabalho.

Faz-se necessário frisar, em um mundo globalizado, a importância do desenvolvimento de pesquisas referentes a ataques a sistemas de utilities, em função de constantes ameaças de investidas ciberterroristas. Segurança da Informação, Ciberataques, Ciberterrorismo e Segurança de Automação industrial estão na pauta dos assuntos que envolvem os mais modernos conceitos tecnológicos e a Internet das Coisas aparece como solução, fornecendo benefícios econômicos e comerciais, ao mesmo tempo em que auxiliam a alavancar a sustentabilidade e conectividades mundiais.

A guisa de conclusão, entendem os autores desse estudo que à medida que as pessoas, bem como as tubulações de água, se conectem à IoT, o mundo será, potencialmente, um lugar melhor.

\section{REFERÊNCIAS BIBLIOGRÁFICAS}

Accenture. (2016). Digital utility transforming for value and growth. Disponivel em: https://www.accenture.com/us- en/ /media/accenture/conversion-assets/ /global/pdf/industries_9/accenture-digital-utilitytransforming-value-growth-inforgraphic.pdf

BCG. (2015). How to jump-start a digital transformation. BCG perspectives. Disponível em: https://www.bcgperspectives.com/content/articles/transformation-large-scale-changetechnology-business-transformation-how-jump-start-digitaltransformation/

Borges, M; Sá, Lucilene Antunes C.M. ; Gomes, H. P. (2004). Utilização de signo monitoramento de avarias em redes de abastecimento de água. In IV Serea - Seminário Hispano-Brasileiro sobre sistemas de abastecimento urbano de água, Joao pessoa. Anais do IV Serea.

Cabezas, J. (2016). Fiware experiences from a hardware company. [s.l: s.n.].

Duarte, R. M. (2010). Geoprocessamento no planejamento urbano. Curitiba: Infogeo.

Fiaschetti, A.; Pietrabissa, A.; Delli Priscoli, F. (2015). Towards manufacturing 2.0: an innovative architecture for the factory of the future. European conference on 
networks and communications, EUCNC, pp. 450-454.

Finesce. seventh framework. The finesce smart energy platform. $\mathrm{n}$. 604677, 2016.

Gartner. (2015). The internet of things is a revolution waiting to happen. disponivel em: http://www.gartner.com/smarterwithgartner/the-internet-ofthings-is-a-revolution-waitingto-happen/

Hompel, M.; Nettstrater, A; Feldhorst, S.; Schier, A. (2012). An intelligent controlling system for greenhouse environment based on the architecture of the internet things. Journal sensor letters.

J-Scoop. digital transformation: focus on the utilities industry. 2016. disponível em: http://www.iscoop.eu/digital-transformation/digital-transformation-focus-on-the-utilities-industry/

Jenkins, Ted. (2016). A better way to manage water with the internet of things. IBM, magazine

Kroll, D. (2006).. Pennwell: 2006. Securing our water supply: proctecting a vulnerable resource.

Luo, H.; Ang, P.; Li, Y.; Xu, F. (2012). An intelligent controlling system for greenhouse environment based on the architecture of the internet things. - Journal Sensor Letters.

Mckinsey. (2016). The digital utility: new opportunities and challenges. disponivel em: http://www.mckinsey.com/industries/electric-power-and-naturalgas/our-insights/the-digital-utility-new-opportunities-and-challenges .

Meola, Andrew. (2016). Lot for utilities: smart water, gas \& electric utilities. Journal Business Insider.

Mounce, S. R. (2015). Cloud based machine learning approaches for leakage assessment and management in smart water networks. Procedia engineering, $v$. 119, pp. 43-52.

Muhammad, S. Y (2015). Classification model for water quality using machine learning techniques. International Journal of software engineering and its applications, v. 9, n. 6, p. 45-52, 2015.

Oren, G.; Stroh, N. Y.(2013). Mathematical model for detection of leakage in domestic water supply systems by reading consumption from an analogue water meter. international journal of environmental science and development, v. 4, n. 4, p. 386. 
Schwab, K. (2016). The fourth industrial revolution. vint research report, p. 1-39, 2016.

Soldato, S. M. (2016). Internet of things applications in future manufacturing. Fitman.

Sun, E; Zang, X; Eli, Z. (2012). The internet os things (iot) and cloud computing (cc). journal safety science.

\section{PARA CITAR ESTE ARTÍCULO:}

Barbosa, A., Costa, C., Texeira, M. y Takeo, S. (2017). Gerenciamento da agua com a internet das coisas (OIT): uma aplicação em plantas de saneamento. Collectivus, Revista de Ciencias Sociales, 4(2), 124-140.

DOI: http://dx.doi.org/10.15648/Coll.2.2017.7

Recibido: 11/03/2017 Aprobado: 14/05/2017 